\title{
Anticompetition Between Laser Modes in Quantum-Dot Laser
}

\author{
Yi-Shin Su, Ding-I Chiang, and Ching-Fuh Lin
}

\begin{abstract}
Anticompetition behavior is observed in a InAs quantum-dot (QD) laser with external wavelength control. Unlike the competition behavior observed in other QD lasers, in this experiment, the laser emission induced by external feedback at the ground state wavelength improves the excited-state emission at $1170 \mathrm{~nm}$ by $13 \mathrm{~dB}$. Such an anticompetition phenomenon is most obvious as the feedback wavelength differs from $1250 \mathrm{~nm}$, the original lasing wavelength, by $15 \mathrm{~nm}$. This anticompetition behavior is explained by the sharing of QDs between the laser emission induced by the feedback and the original laser oscillation.
\end{abstract}

Index Terms-Carrier dynamics, nonlinear optical device, quantum dots (QDs), semiconductor lasers.

\section{INTRODUCTION}

Q UANTUM-DOT (QD) lasers are attractive because they exhibit excellent properties such as extremely low operation current density, high thermal stability, and so on [1]-[4]. Although high-quality QDs can be grown by the Stranski-Krastanov method, there is inevitable variation in the dot sizes and thus the quantized energy levels. The inhomogeneous broadening of the gain spectrum due to variation in the dot size provides complex dynamical behaviors to be explored. In all-optical networks, the application or the elimination of the gain competition phenomenon is of special importance. Using QDs as the gain media, optical amplifiers can achieve broad bandwidth and low crosstalks [5]-[8]. This property is also preferred when amplification of mode-locked pulses with broad spectrum is considered [9]. On the other hand, QD devices still exhibit strong localized spectral hole burning due to homogeneous broadening of each QD [7]. This special combination of weak global competition and strong local competition is suitable for single-device all-optical multichannel switches [10], [11]. In addition to the competition behaviors, QD lasers can have anticompetition behaviors by the mechanism of optical pumping [12]. In this work, we found another mechanism that

Manuscript received December 14, 2006; revised March 30, 2007. This work was supported in part by the National Science Council (NSC) of R.O.C. under Grant NSC 95-2120-M-002-001 and Grant NSC 95-2112-M-002-004.

Y.-S. Su and D.-I Chiang are with the Graduate Institute of Electro-Optical Engineering, National Taiwan University, Taipei, 10617 Taiwan, R.O.C. (e-mail: yssu@ntu.edu.tw).

C.-F. Lin is with the Graduate Institute of Electro-Optical Engineering and Department of Electrical Engineering, National Taiwan University, Taipei, 10617 Taiwan, R.O.C., and also with the Graduate Institute of Electronics Engineering, National Taiwan University, Taipei, 10617 Taiwan, R.O.C. (e-mail: cfling@cc.ee.ntu.edu.tw).

Color versions of one or more of the figures in this letter are available online at http://ieeexplore.ieee.org.

Digital Object Identifier 10.1109/LPT.2007.897560

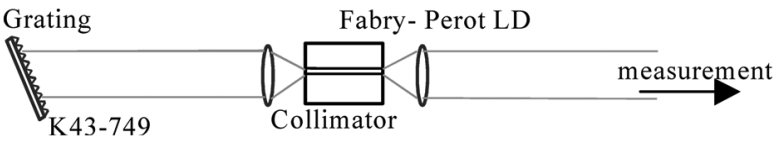

Fig. 1. External cavity laser setup.

leads to anticompetition behavior. With this new mechanism, the laser emission at the ground state induced by grating feedback enhances the emission of the excited states. This behavior is different from the competition behavior normally observed in QD amplifiers, and is also different from the anticompetition previously observed in the QD two-wavelength laser [12].

\section{EXPERIMENETAL SETUP AND QD LASER CHARACTERISTICS}

This anticompetition behavior is observed in a QD laser under the experimental setup shown in Fig. 1. This laser chip has InAs QDs as the gain material. The QDs are embedded in the InGaAs-GaAs quantum well. The substrate is GaAs. Two types of QD layers with different size distributions are used together to enhance the effect of inhomogeneous broadening. One type of QD layer has the center emission wavelength at $1.24 \mu \mathrm{m}$. The other has the center emission wavelength at $1.28 \mu \mathrm{m}$. The fabricated laser chip has $497-\mu \mathrm{m}$ cavity length and $4-\mu \mathrm{m}$ waveguide width. Without using the external cavity, this Fabry-Pérot laser diode has the threshold current of $18.1 \mathrm{~mA}$. The ground-state laser emission covers many Fabry-Pérot modes with a peak wavelength at $1250 \mathrm{~nm}$. The amplified spontaneous emission of the excited states is observed to be between 1170 and $1180 \mathrm{~nm}$.

In the setup, the light emitted from both facets is collimated using two collimators. At one side of the laser diode, a grating is used to provide control on the emission wavelength of the external-cavity laser. The emission wavelength controlled by the grating is called the feedback wavelength for the purpose of description simplicity later. In the tunable range of this laser, the reflection efficiency of this grating is above $60 \%$. The emission from the other side is used for spectral analysis.

\section{ANTICOMPETITION BEHAVIOR}

In the grating-controlled external-cavity laser, it is well known that the lasing characteristics are influenced by the feedback wavelength. Here we further discover that the laser emission at the ground-state wavelength induced by the external feedback does not necessarily compete with the emission at the excited-state wavelength, such as the case when the feedback wavelength is at $1265 \mathrm{~nm}$. Fig. 2(a) shows the emission spectrum of this device at $64 \mathrm{~mA}$ without the external cavity, while Fig. 2(b) is the emission spectrum with the feedback wavelength at $1265 \mathrm{~nm}$. When the grating feedback is applied 


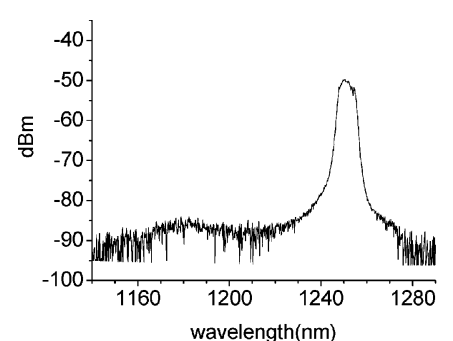

(a)

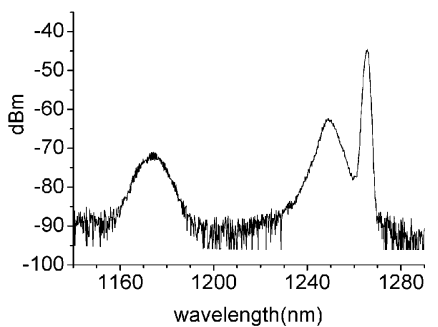

(b)
Fig. 2. Emission spectra of this laser at $64 \mathrm{~mA}$ : (a) no external feedback; (b) external feedback at $1265 \mathrm{~nm}$.

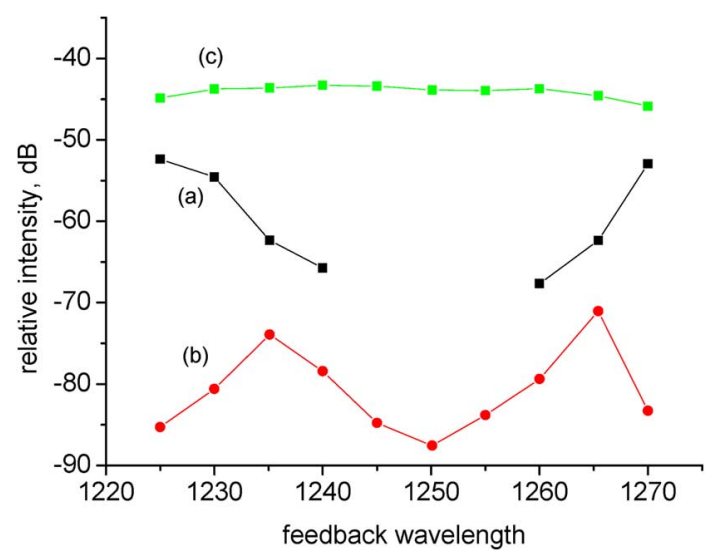

Fig. 3. Amplitude of each peak in the emission spectra at $64 \mathrm{~mA}$. (a) Original laser emission at $1250 \mathrm{~nm}$. (b) Excited-state emission at $1170 \mathrm{~nm}$. (c) Laser emission at feedback wavelength. Without feedback, the peak intensity of the original laser emission at $1250 \mathrm{~nm}$ is $-49.72 \mathrm{~dB}$, and the peak excited-state emission intensity is $-84.08 \mathrm{~dB}$.

at $1265 \mathrm{~nm}$, the external cavity induces a new laser emission at this feedback wavelength. At the same time, the peak intensity at the original laser emission, $1250 \mathrm{~nm}$, drops by $12.6 \mathrm{~dB}$, but the emission spectrum corresponding to the excited states increases significantly by $13 \mathrm{~dB}$. The spectral changes are clearly observed by comparing Figs. 2(a) and (b). Therefore, the increase of laser intensity at $1265 \mathrm{~nm}$ results in the increase of emission intensity from the excited states, the anticompetition phenomenon.

This anticompetition phenomenon is dependent on the feedback wavelength. The peak intensity of the original ground-state laser emission and the excited-state emission are plotted against the feedback wavelength as curves (a) and (b), respectively, in Fig. 3. The intensity of the laser emission at the feedback wavelength is also shown in Fig. 3 [curve (c)]. The injection current for the measurements shown in Fig. 3 is $64 \mathrm{~mA}$. In Fig. 3, the laser emission intensity at the feedback wavelength, curve (c), only varies slightly, less than $3 \mathrm{~dB}$ in the tuning range, while the emission intensity of excited states, curve (b), varies for more than $16 \mathrm{~dB}$. The excited-state emission intensity has maximum when the feedback wavelength is at 1235 and $1265 \mathrm{~nm}$, which are both $15 \mathrm{~nm}$ away from the original laser emission of the ground states. In both cases, the peak emission intensity of the excited states is $13 \mathrm{~dB}$ higher than the value without feedback. When the feedback wavelength moves further away

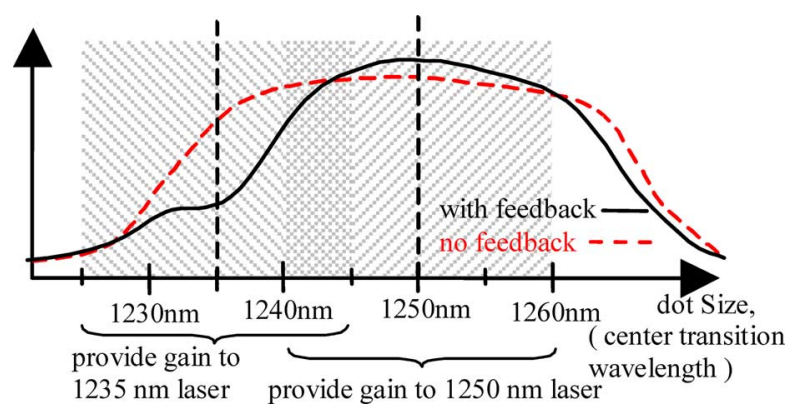

Fig. 4. Carrier distribution of the ground states under the influence of external feedback. Solid line is the case when feedback is applied at $1235 \mathrm{~nm}$. Dashed line is the case without external feedback.

from $1250 \mathrm{~nm}$, the peak intensity of the excited-state emission decreases and the intensity of the original laser emission at $1250 \mathrm{~nm}$ recovers. The effect of external feedback on the emission property is reduced. On the other hand, when the feedback wavelength shifts from 1235 or $1265 \mathrm{~nm}$ toward $1250 \mathrm{~nm}$, the peak intensity of the excited-state emission decreases and finally drops to lower than the value without feedback. The original laser emission at $1250 \mathrm{~nm}$ decreases, too. For the feedback wavelength at $1250 \mathrm{~nm}$, the external feedback is observed to compete with the original emissions of both the excited states and the ground states.

\section{DISCUSSION}

This peculiar behavior can be explained by the interaction between QDs of different transition wavelengths. The gain spectrum of each single QD has a finite linewidth. This linewidth measured by the gain competition effect ranges from 12 to $24 \mathrm{~nm}$ [7], [11]. For simplicity of discussion, let us assume the homogeneous-broadened gain spectrum of each QD covers a 20-nm spectral range. By this assumption, the laser emission at 1250-nm wavelength is contributed by QDs with transition wavelength between 1240 and $1260 \mathrm{~nm}$. Considering the case with another laser emission at $1235 \mathrm{~nm}$ induced by grating feedback, the gain at $1235 \mathrm{~nm}$ is contributed by QDs with transition wavelength from 1225 to $1245 \mathrm{~nm}$. The QDs with a transition wavelength between 1240 and $1245 \mathrm{~nm}$ provide gain to both emissions at 1235 and $1250 \mathrm{~nm}$, while the QDs with a transition wavelength between 1245 and $1260 \mathrm{~nm}$ provide gain to the emission at $1250 \mathrm{~nm}$ only. As illustrated in Fig. 4, the carrier population in QDs with a transition wavelength from 1240 to $1245 \mathrm{~nm}$ decreases due to the stimulated emission induced by the 1235-nm laser emission. Because these QDs also provide gain to the original laser emission at $1250 \mathrm{~nm}$, the 1250-nm laser intensity decreases.

In QDs with a transition wavelength longer than $1260 \mathrm{~nm}$, the carriers do not provide gain for laser emission at $1235 \mathrm{~nm}$, but the carrier population should also decrease by a small amount due to interdot carrier redistribution. However, the situation is different for QDs with transition wavelengths from 1245 to $1260 \mathrm{~nm}$. The 1250-nm laser induces significant stimulated recombination in these QDs. The stimulated recombination rate is proportional to the product of the laser intensity at $1250 \mathrm{~nm}$ and the provided gain. When the laser intensity at $1250 \mathrm{~nm}$ decreases, the stimulated recombination rate in these 
QDs decreases. Thus, in the QDs, which contribute gain to $1250 \mathrm{~nm}$ only, extra carriers accumulate in the ground states and the number of empty ground states decreases, as illustrated in Fig. 4. Because of the Pauli exclusion principle, excited-state carriers can only fall to empty sites of the ground states. Thus, the rate of excited-state carriers falling to the ground states decreases. The excited-state carriers in these QDs also increase and additional emissions can be observed at the corresponding wavelength, $1170 \mathrm{~nm}$. This is the observed anticompetition effect in this experiment.

The above model also explains the reduction of this anticompetition effect when the feedback wavelength moves toward or away from 1235 or $1265 \mathrm{~nm}$. As the separation between the feedback wavelength and $1250 \mathrm{~nm}$ increases, the amount of QDs shared by both wavelengths decrease. Thus, less QDs contributing gain at $1250 \mathrm{~nm}$ are affected by the external feedback. The laser emission at $1250 \mathrm{~nm}$ recovers and the carrier population of the 1250-nm QDs decreases again.

From the above arguments, moving the feedback wavelength closer to $1250 \mathrm{~nm}$ is expected to increase the anticompetition effect. However, the emission intensity of the excited states decreases when the feedback wavelength moves to less than 10 from $1250 \mathrm{~nm}$. The reason is as follows. When the feedback wavelength lies within the homogeneously broadened gain spectrum of the QDs with 1250-nm transition wavelength, the laser emission at the feedback wavelength can also induce stimulated recombination in these 1250-nm QDs. This results in strong carrier competition. Ground-state carriers in these 1250-nm QDs will decrease due to the stimulated recombination induced by the laser emission at the feedback wavelength. The anticompetition effect is balanced by the usual competition effect.

Although we assume the homogeneously broadened gain spectrum to cover $20-\mathrm{nm}$ spectral rang, a smaller value of homogeneous-broadening linewidth can also explain the experiment well. The reason is because the competition effect due to the homogeneous broadening is not limited to the full-width at half-maximum (FWHM) range. When the feedback wavelength is close to $1250 \mathrm{~nm}$, the competition effect is strong. It gradually decreases as the feedback wavelength moves away from $1250 \mathrm{~nm}$. In the experiment, the competition effect is more significant than the anticompetition effect when the feedback wavelength is less than $+/-10$ from $1250 \mathrm{~nm}$. If this $+/-10-n m$ wavelength span corresponds to the $90 \%$ width of the homogeneously broadened gain spectrum, then the homogeneous-broadening linewidth should have 9-meV FWHM. Whether this $+/-10$-nm wavelength span is the $50 \%$ width (16-meV homogeneous broadening) or the $90 \%$ width (9-meV homogeneous broadening) depends on the relative strengths of the two counteracting effects, the competition and the anticompetition.

\section{CONCLUSION}

A new type of anticompetition behavior was observed in the QD external-cavity tunable laser. In this investigation, we discovered that the laser emission at the ground state induced by grating feedback enhances the emission of the excited states. This anticompetition phenomenon is dependent on the feedback wavelength. The increment in the excited-state emission intensity is maximum when the feedback wavelength is at 1235 and $1265 \mathrm{~nm}$, which are both $15 \mathrm{~nm}$ away from the original laser emission, $1250 \mathrm{~nm}$. The anticompetition behavior is explained by the sharing of QDs between the laser emission induced by the feedback and the original laser oscillation. When the feedback wavelength moves away from $1250 \mathrm{~nm}$, the number of shared QDs decreases, leading to the reduced anticompetition. On the other hand, when the feedback wavelength moves toward $1250 \mathrm{~nm}$, the competition between the laser emission induced by the feedback and the original laser oscillation increases as a result of homogeneous broadening. It also leads to the apparent reduction in the anticompetition.

\section{REFERENCES}

[1] N. Kirstaedter, N. N. Ledentsov, M. Grundmann, D. Bimberg, V. M Ustinov, S. S. Ruvimov, M. V. Maximov, P. S. Kop'ev, Z. I. Alferov, U. Richter, P. Werner, U. Goesele, and J. Heydenreich, "Low threshold, large $T_{0}$ injection laser emission from InGaAs quantum dots," Electron. Lett., vol. 30, no. 17, pp. 1416-1417, Aug. 1994.

[2] M. Sugawara, Self-Assembled InGaAs/GaAs Quantum Dots, Semiconductor and Semimetals. New York: Academic, 1999, vol. 60.

[3] G. T. Liu, A. Stintz, H. Li, K. J. Malloy, and L. F. Lester, "Extremely low room-temperature threshold current density diode lasers using InAs dots in $\mathrm{In}_{0.15} \mathrm{Ga}_{0.85}$ As quantum well," Electron. Lett., vol. 35, no. 14, pp. 1163-1165, Jul. 1999.

[4] P. G. Eliseev, H. Li, A. Stintz, G. T. Liu, T. C. Newell, K. J. Malloy, and L. F. Lester, "Transition dipole moment of InAs/InGaAs quantum dots from experiments on ultralow-threshold laser diodes," Appl. Phys. Lett., vol. 77, no. 2, pp. 262-264, Jul. 2000.

[5] P. M. Varangis, H. Li, G. T. Liu, T. C. Newell, A. Stintz, B. Fuchs, K. J. Malloy, and L. F. Lester, "Low-threshold quantum dot lasers with $201 \mathrm{~nm}$ tuning range," Electron. Lett., vol. 36, no. 18, pp. 1544-1545, Aug. 2000.

[6] T. Akiyama, M. Ekawa, M. Sugawara, K. Kawaguchi, H. Sudo, A. Kuramata, H. Ebe, K. Morito, and Y. Arakawa, "An ultrawide-band semiconductor optical amplifier having an extremely high penalty-free output power of $23 \mathrm{dBm}$ achieved with quantum dots," IEEE Photon. Technol. Lett., vol. 17, no. 8, pp. 1614-1616, Aug. 2005.

[7] P. Eliseev, H. Li, A. Stintz, G. T. Liu, T. C. Newell, K. J. Malloy, and L. F. Lester, "Tunable grating-coupled laser oscillation and spectral hole burning in an InAs quantum-dot laser diode," IEEE J. Quantum Electron., vol. 36, no. 4, pp. 479-485, Apr. 2000.

[8] R. Alizon, D. Hadass, V. Mikhelashvili, G. Eisenstein, R. Schwertberger, A. Somers, J. P. Reithmaier, A. Forchel, M. Calligaro, S. Bansropun, and M. Krakowski, "Multiple wavelength amplification in wide band high power $1550 \mathrm{~nm}$ quantum dash optical amplifier," Electron. Lett., vol. 40, no. 12, pp. 760-761, Jun. 2004.

[9] E. U. Rafailov, P. Loza-Alvarez, W. Sibbett, G. S. Sokolovskii, D. A. Livshits, A. E. Zhukov, and V. M. Ustinov, "Amplification of femtosecond pulses over by $18 \mathrm{~dB}$ in a quantum-dot semiconductor optical amplifier," IEEE Photon. Technol. Lett., vol. 15, no. 19, pp. 1023-1025, Aug. 2003.

[10] D. Alexander, J. S. Deogun, and H. S. Hamza, "Quantum dot based technology for multiple wavelength conversion," in Proc. LEOS 2005, 18th Annual Meeting of IEEE, Oct. 2005, pp. 126-127.

[11] T. Akiyama, H. Kuwatsuka, T. Simoyama, Y. Nakata, K. Mukai, M. Sugawara, O. Wada, and H. Ishikawa, "Application of spectral-hole burning in the inhomogeneously broadened gain of self-assembled quantum dots to a multiwavelength-channel nonlinear optical device," IEEE Photon. Technol. Lett., vol. 12, no. 10, pp. 1301-1303, Aug. 2000.

[12] Y. S. Su and C. F. Lin, "Anticompetition of laser modes in quantum dot lasers," in Proc. CLEO 2005, May 2005, vol. 3, pp. 1617-1673, Paper CThH5. 